This item was submitted to Loughborough's Research Repository by the author.

Items in Figshare are protected by copyright, with all rights reserved, unless otherwise indicated.

\title{
The effects of high-intensity interval training on glucose regulation and insulin resistance: a meta-analysis
}

\section{PLEASE CITE THE PUBLISHED VERSION}

http://dx.doi.org/10.1111/obr.12317

\section{PUBLISHER}

(c) Wiley

\section{VERSION}

AM (Accepted Manuscript)

\section{PUBLISHER STATEMENT}

This work is made available according to the conditions of the Creative Commons Attribution-NonCommercialNoDerivatives 4.0 International (CC BY-NC-ND 4.0) licence. Full details of this licence are available at: https://creativecommons.org/licenses/by-nc-nd/4.0/

\section{LICENCE}

CC BY-NC-ND 4.0

\section{REPOSITORY RECORD}

Jelleyman, C., Gary O'Donovan, TY Yates, L.J. Gray, James King, Kamlesh Khunti, and Melanie J. Davies. 2015. "The Effects of High-intensity Interval Training on Glucose Regulation and Insulin Resistance: A Metaanalysis". Loughborough University. https://hdl.handle.net/2134/19054. 
The effects of high-intensity interval training on glucose regulation and insulin resistance: a meta-analysis

Charlotte Jelleyman ${ }^{1,2}$, Thomas Yates ${ }^{1,2}$, Gary O’Donovan ${ }^{1}$, Laura J Gray ${ }^{3}$, James A King ${ }^{2,4}$, Kamlesh Khunti $^{1,5}$ \& Melanie J Davies ${ }^{1,2}$

1. Diabetes Research Centre, University of Leicester, Leicester, UK

2. NIHR Leicester-Loughborough Diet, Lifestyle and Physical Activity Biomedical Research Unit, UK

3. Department of Health Sciences, University of Leicester, Leicester, UK

4. School of Sport, Exercise and Health Sciences, Loughborough University, Loughborough, UK

5. NIHR Collaboration for Leadership in Applied Health Research and Care - East Midlands (NIHR CLAHRC - EM)

* Present affiliation correspondence: Charlotte Jelleyman, Diabetes Research Centre, Leicester General Hospital, Leicester, LE5 4PW, UK; cj136@le.ac.uk, +44 (0)116 2584394

\section{Running Title}

The effects of HIIT on metabolic health

\section{Declaration of interests}

The authors declare that there is no conflict of interest associated with this manuscript.

\section{Acknowledgements}

The authors acknowledge support from the National Institute for Health Research Collaboration for Leadership in Applied Health Research and Care - East Midlands (NIHR CLAHRC - EM), the Leicester Clinical Trials Unit and the NIHR Leicester-Loughborough Diet, Lifestyle and Physical Activity Biomedical Research Unit, which is a partnership between University Hospitals of Leicester NHS Trust, Loughborough University and the University of Leicester. 


\begin{abstract}
The aim of this meta-analysis was to quantify the effects of high-intensity interval training (HIIT) on markers of glucose regulation and insulin resistance compared to control conditions (CON) or continuous training $(\mathrm{CT})$. Databases were searched for HIIT interventions based on the inclusion criteria: training $\geq 2$ weeks, adult participants, and outcome measurements that included insulin resistance, fasting glucose, HbA1c or fasting insulin. Dual interventions and participants with type 1 diabetes were excluded. Fifty studies were included. There was a reduction in insulin resistance following HIIT compared to both CON \& $\mathrm{CT}$, (HIIT vs. CON: standardised mean difference (SMD) $=-0.49$, confidence intervals (CI) -0.87 to -0.12 , $\mathrm{p}=0.009$; CT: $\mathrm{SMD}=-0.35,-0.68$ to $-0.02, \mathrm{p}=0.036)$. Compared to CON, HbA1c decreased by $0.19 \%(-0.36$ to $-0.03, \mathrm{p}=0.021)$ and body weight decreased by $1.3 \mathrm{~kg}(-1.9$ to $-0.7, \mathrm{p}<0.001)$. There were no statistically significant differences between groups in other outcomes overall. However, participants with or at risk of Type 2 diabetes experienced reductions in fasting glucose $\left(-0.92 \mathrm{mmol} . \mathrm{L}^{-1},-1.22\right.$ to $\left.-0.62, \mathrm{p}<0.001\right)$ compared to CON. HIIT appears effective at improving metabolic health, particularly in those at risk of or with Type 2 diabetes. Larger randomised controlled trials of longer duration than those included in this meta-analysis are required to confirm these results.
\end{abstract}




\section{Introduction}

Obesity and Type 2 diabetes are inextricably linked with over $80 \%$ of people with Type 2 diabetes classed as overweight or obese based on BMI thresholds. ${ }^{1}$ Diet and physical activity interventions are the cornerstones for management of both conditions. However, whilst effects of exercise on Type 2 diabetes and insulin sensitivity are well established, ${ }^{2-4}$ the effects on weight regulation are more controversial. ${ }^{5,6}$ The prevailing recommendation for meaningful improvements in cardiorespiratory fitness and metabolic health to occur in adults is engaging in a minimum of 150 minutes of moderate-intensity or 75 minutes of vigorous-intensity physical activity per week, accumulated in bouts of 10 minutes or more. ${ }^{7-9}$ The guidelines for weight loss are greater; suggesting that 200-300 minutes per week is required for long term reductions. ${ }^{10}$ Given that less than $50 \%$ of the population in industrialised societies, ${ }^{11}$ with estimates falling to as low as $5 \%$ when objective measures of physical activity are employed, ${ }^{12,13}$ meet the shorter physical activity recommendations for health, it is becoming more important to elucidate what is the minimum amount of physical activity required to promote health benefits. This notion is supported by findings from surveys investigating perceived barriers to participation in physical activity which consistently highlight "lack of time" as a common barrier for not being more active, a finding applicable to the general population ${ }^{14,15}$ as well as those with Type 2 diabetes. ${ }^{16}$ High-intensity interval training (HIIT) has been proposed as a time-efficient exercise intervention that may bring about similar benefits as moderate-intensity aerobic exercise. ${ }^{17,18}$ Sprint interval training (SIT) using the Wingate protocol is a well-defined form of HIIT involving just three minutes of activity per session not including warm-up or cool-down. ${ }^{19}$ Although this version of HIIT has been shown to improve fitness in a variety of populations, ${ }^{20-22}$ the repeated maximal efforts this protocol requires may limit practicality for sedentary individuals. ${ }^{23}$ As such, protocols using longer, submaximal intervals have been developed, a form of HIIT described as "aerobic interval training”. ${ }^{24,25}$ For the purpose of this review, any form of interval training that incorporates high-intensity exercise within or above the range categorised as vigorous (64-90\% $\mathrm{VO}_{2 \max }$ or $77-95 \% \mathrm{HR}_{\max }$ ) in the American College of Sports Medicine guidelines ${ }^{26}$ shall be collectively referred to as HIIT (i.e. sprint interval training, aerobic interval training).

While HIIT tends to have a potent effect on cardiorespiratory fitness in a variety of populations ${ }^{27-29}$, benefits to obesity and markers of metabolic health, such as glucose regulation and insulin sensitivity are less well defined. One narrative review concluded that despite a reduction in total work volume, HIIT has positive effects on blood glucose control and insulin sensitivity compared to continuous exercise. ${ }^{30}$ This literature review was limited as it did not provide quantification of the effect of HIIT on metabolic health outcomes. Nor did it assess the impact of varying HIIT characteristics. The aim of this systematic review was therefore to quantify the impact of HIIT on glucose and insulin regulation, body weight and cardiorespiratory fitness compared to control conditions (CON) or continuous exercise training (CT) using meta-analysis. A secondary aim was to assess whether observed metabolic changes were mediated by characteristics of the training protocol (i.e. interval intensity, training volume) or concurrent changes in participant physiology (e.g. cardiorespiratory fitness, body weight). 


\section{Methods}

This meta-analysis has been reported according to the preferred reporting items for systematic reviews and meta-analyses (PRISMA) guidelines. ${ }^{31}$ See Supplement 1 for the checklist.

\section{Search strategy and inclusion criteria}

Medline (1946-13/03/2015), Embase (1970-13/03/2015) and SportDiscuss (1953-30/03/2015) were searched for HIIT intervention studies that reported a measure of glycaemic control. There is no universal definition of HIIT, therefore, based on a brief overview of the literature, we applied the following criteria to our search: at least two bouts of vigorous- or higher intensity exercise ${ }^{26}$ interspersed with periods of lower intensity exercise or complete rest. "High-intensity interval training” is not a MeSH term therefore words and phrases commonly used to describe HIIT were searched in titles and abstracts using the following search terms: "high-intensity interval”, “aerobic interval” and "sprint interval”. These were then combined with the following terms using Boolean commands: intermittent, Wingate, supramaximal, exercise, training, programme, glucose, insulin, glycaemic, and HbA1c. Wildcards: *; ? and \$ were used so that both English and American spellings would be returned. Supplement 2 gives a detailed description of the search strategy. Titles and abstracts of returned articles were evaluated based on the following inclusion criteria: human participants aged 18 years or over, participants receiving a HIIT intervention, and at least one measure of glycaemic control defined as: HbA1c, fasting glucose, fasting insulin, postprandial or post-challenge glucose response, or any measure of insulin resistance assessed pre- and post-intervention. HIIT had to be prescribed at least three times per week for two weeks. Two weeks was deemed the minimum period needed to show training adaptations; defined as a temporary or extended change in structure or function that results from performing repeated bouts of exercise and that is independent of the immediate or short-term effects produced by a single bout of exercise. ${ }^{32}$ Both controlled and uncontrolled studies were included. Articles were excluded if HIIT was prescribed in combination with another intervention e.g. diet restriction; resistance training, if participants had diagnosed Type 1 Diabetes (studies of people with Type 2 diabetes were included), or if medication had been altered throughout the intervention. Abstracts, case reports, observational studies and studies not published in English were also excluded.

\section{Risk of bias and study quality}

Risk of bias was evaluated based on the PRISMA recommendations ${ }^{31}$ which suggest assessing randomised control trial quality using the Cochrane risk of bias tool. ${ }^{33}$ This tool consists of five items that have been shown to have an effect on biasing the results of an intervention. Studies with control groups were checked for random sequence generation, allocation concealment, blinding, participants lost to follow-up, and whether an intention-to-treat analysis had been performed. A score of one point was given for each item fulfilled such that studies could score a maximum of five points. Studies without clear descriptions of these processes were considered not to have satisfied these criteria. Uncontrolled trials were not assessed.

\section{Data extraction and synthesis}


Reviewers were not blinded to study authors, institutions, or manuscript journals. If the abstract was considered to be relevant to the review, or did not contain enough information regarding the inclusion or exclusion criteria, full-texts were retrieved for further evaluation. References included in identified studies and previous reviews or commentaries were also hand searched. Where there was uncertainty by the first reviewer regarding appropriate studies, the full text was obtained and a second reviewer (TY) approached for discussion. If evidence of participant repetition was evident participants were only included once, however if necessary, multiple articles were used to obtain all required data.

If, according to the methodology relevant measurements had been taken but the results not reported, or values had been presented in figures, authors were contacted and asked to provide the missing data. When no reply was received the study/outcome was either omitted from the analysis ${ }^{34}$ or values estimated from figures. ${ }^{35-37}$ Where only pre and post-intervention data were presented, change data were imputed based on guidelines from the Cochrane Handbook for Systematic Reviews of Interventions. ${ }^{38}$

A data extraction form was created and data regarding participant characteristics and disease status, protocol specifics, CT interventions, markers of glucose regulation, insulin resistance, $\mathrm{VO}_{2 \max }$, body composition and compliance, attrition, and adverse events were entered independently by two reviewers (CJ and GO).

Discrepancies were resolved by consensus or by a third reviewer (TY). A number of studies reported results from both acute (up to $48 \mathrm{~h}$ ) and longer term (72h) blood samples. If this was the case, the $72 \mathrm{~h}$ reading was included in the analysis. Since the study by Lunt et al. ${ }^{39}$ had two HIIT groups and a CT group, as per the Cochrane guidelines, the number of participants in the CT group was halved so that pairwise comparisons between HIIT and continuous exercise could be made for each HIIT protocol. One study compared two HIIT groups and these were entered as separate, uncontrolled trials. ${ }^{40}$

All models of insulin sensitivity were expressed as insulin resistance to account for the directional effect of exercise since a beneficial effect would increase sensitivity and decrease resistance. HOMA-IS\% values were inverted (100/HOMA-IS\% $)^{41}$, and change scores for other models of insulin sensitivity $(n=9,20 \%)$ were multiplied by -1 .

\section{Statistical analysis}

Stata v.13 (StataCorp. 2013. Stata Statistical Software: Release 13. College Station, TX, USA) was used to conduct the meta-analyses. Pairwise comparisons comparing the effect of HIIT on glucose/insulin parameters and $\mathrm{VO}_{2 \max }$, to that of either $\mathrm{CT}$ or $\mathrm{CON}$ were carried out on studies that had two or more groups. In keeping with other exercise-related meta-analyses of continuous outcomes, ${ }^{3,42}$ and following best practice, ${ }^{38}$ weighted mean differences were calculated in the pairwise comparisons for glucose/insulin parameters and $\mathrm{VO}_{2 \max }$. Standardised mean differences were used to account for the different measures of insulin resistance.

When studies had a HIIT group only, within group intervention effect sizes were calculated to estimate the change from baseline. All studies with a control group were included in both the between and within group 
comparisons. Since this within group comparison is based on unstandardised data, only HOMA-derived insulin resistance measures could be assessed in this analysis.

Participants were stratified by health characteristics based on the descriptions given by each included study as follows: healthy (well-trained/recreationally active/sedentary); overweight/obese; metabolic syndrome (MetS)/Type 2 diabetes; with another chronic disease. Data were presented according to disease status.

We also performed two sensitivity analyses for insulin resistance: 1) using HOMA scores only to determine whether results were attenuated when more sensitive measures of peripheral insulin resistance were removed and 2) by the length of time elapsed before blood was sampled following the last training session i.e. $<24 \mathrm{~h}$, $\geq 24 \mathrm{~h}$ and $<72 \mathrm{~h}$ or $\geq 72 \mathrm{~h}$; the latter analysis was undertaken on the within group analysis only due to lack of data for the between group comparisons.

Random effects models using Cohen's $d$ were carried out to account for the differences in study protocol and duration. Statistical heterogeneity of the treatment effect among studies was assessed using the chi-squared test. A threshold $\alpha$ value of $<0.05$ was considered statistically significant and an $I^{2}$ test with values greater than $50 \%$ were indicative of high heterogeneity.

\section{Publication bias}

Publication bias based on reporting of the main outcomes was assessed using a contour-enhanced funnel plot of each trial's effect size against the standard error. ${ }^{43}$ Funnel plot asymmetry was assessed by visual interpretation. If publication bias was apparent, Begg \& Egger tests were used as a secondary determinant. ${ }^{44}$ ${ }^{45}$ Significant publication bias was deemed apparent if $\mathrm{p}<0.1$.

\section{Meta-regression}

Where significant results were found, meta-regression was performed in an attempt to determine whether baseline levels, exercise volume variables and changes to body weight and $\mathrm{VO}_{2 \max }$ mediated observed changes.

Interval intensity and weekly high-intensity exercise duration and total training period (weeks) were deemed the most relevant components of HIIT protocols. Where possible, using regression equations derived from early work ${ }^{46,47}$ we converted interval intensity to a percentage of $\mathrm{VO}_{2 \max }$ in order to be able to directly compare exercise prescriptions. High-intensity exercise duration was estimated by multiplying the number of high-intensity intervals $\mathrm{x}$ interval length $\mathrm{x}$ the number of sessions per week and controlled for intensity and the number of weeks the study was run.

Change in body weight and cardiorespiratory fitness were also entered into the regression given their association with the primary outcomes. ${ }^{48,} 49$

For within group regression, change summary data were used as the dependent variable and were weighted by the standard error. In studies with a control group, the dependent variable was the mean difference calculated from the pairwise comparison, with each study weighted by the standard error of its effect size. 


\section{Results}

\section{Studies retrieved}

Study selection flow is presented in Figure 1. The initial searches returned a total of 6209 articles (Medline n=3569, Embase n=1933, SportsDiscuss n=707), of which 4523 were original articles. Titles and abstracts of returned articles were searched for suitability leading to the retrieval of 317 full-texts. Of these, 263 did not fulfil the inclusion criteria and four were excluded due to the nature of the methods used. The total number of papers included in the analysis was 50, described in Table 1. Fourteen (28\%) studies did not have a control group and were therefore only included in the within group analyses. Of the 36 (72\%) controlled trials 14 (30\%) had a CT group, 9 (18\%) a CON group, 11 (22\%) had both, one (2\%) had two HIIT groups and a CT group and one (2\%) compared two HIIT groups.

\section{Study quality and risk of bias}

The 36 controlled trials were assessed for risk of bias. The median quality score was 1/5 (see Table S1). Of the included studies 13/36 (36\%) presented adequate sequence generation, nine (25\%) reported allocation concealment and 11 (31\%) blinded where possible. It was unclear in three (8\%) studies how many participants were lost to follow-up and five (14\%) used the intention-to-treat principle for statistical analysis.

\section{Publication bias}

Visual interpretation of funnel plots suggested limited publication bias and as such no statistical adjustment was made. See Supplement 3 for figures.

\section{Heterogeneity}

Heterogeneity statistics are presented in Table $1 . I^{2}$ values were generally high, with all the within group comparisons indicative of wide heterogeneity (mean score $=89.1 \%$ ). Controlled trials scored lower, with some showing homogenous statistics (mean $\mathrm{CON}=49.2 \%$; $\mathrm{CT}=31.3 \%$ ).

\section{Participants}

There were a total of 2033 participants included in the analysis, of which 1383 (68\%) underwent a HIIT intervention. Participants were aged 21-68 years and spanned a wide range of health and disease characteristics; from well-trained individuals $(n=61,3 \%)$ through recreationally active $(n=895,44 \%)$, sedentary but otherwise healthy ( $\mathrm{n}=86,4 \%)$, overweight/obese ( $\mathrm{n}=230,11 \%)$, with metabolic syndrome ( $\mathrm{n}=157,8 \%$ ), Type 2 diabetes ( $\mathrm{n}=143,7 \%$ ) or with another chronic disease (e.g. cancer, heart failure; $\mathrm{n}=461$, 23\%). For subgroup analysis we stratified participants by disease status; healthy, $n=1042$ (51\%), overweight/obese $n=230$ (11\%), metabolic syndrome (MetS)/Type 2 diabetes $n=300$ (15\%) and other chronic disease $n=461(23 \%)$.

\section{Overview of exercise interventions}


Exercise interventions are described briefly in Table S2. Study protocols varied widely between both HIIT and CT interventions. HIIT interventions included aerobic interval training (e.g. ${ }^{39,50,51}$ ), sprint interval training (e.g. ${ }^{52-54}$ ), and high-intensity interval training (e.g. ${ }^{25,40,55}$ ). The number (range 2 - >60), duration (range 4s-5min) and intensity (range $65 \% \mathrm{VO}_{2 \max }$ - Wingate effort) of "high-intensity" intervals, as well as duration (range $12 \mathrm{~s}-5 \mathrm{~min}$ ) and intensity (range from complete rest- $70 \% \mathrm{HR}_{\max }$ ) of recovery intervals varied widely between studies. Exercise session duration (mean 34 mins, range 10-60 mins) total training volume (range 8-5040 mins) and total length of intervention (range 2- 16 weeks) also varied widely between studies. Not all studies reported how the continuous training intervention had been selected, although some were energy matched to HIIT (e.g. ${ }^{56-58}$ ) or based on the global recommendations for moderate intensity exercise (e.g. ${ }^{59,60}$ ). Continuous training ranged from $30-120$ min per session at intensities between $55 \% \mathrm{VO}_{2 \max } / \mathrm{HR}_{\max }$ to $80 \% \mathrm{HR}_{\max }$.

\section{Training modalities}

In most cases HIIT was carried out in an exercise laboratory supervised by an investigator or trained exercise physiologist. Three studies investigated the practicality of home-based HIIT interventions. ${ }^{61-63}$ An exercise bike was used in 26 (52\%) studies, 15 (30\%) used a treadmill, one (2\%) an athletics track ${ }^{64}$ and six (12\%) a free-living walking environment. ${ }^{39,50,61,62,65}$ Two (4\%) studies allowed participants to choose between treadmill and exercise bike throughout the intervention. ${ }^{55,66}$

\section{Compliance, attrition and adverse events}

Adherence to the intervention was reported by 20 (40\%) studies and was $90 \pm 11 \%$ of exercise sessions. Minimum adherence to be included in analysis was specified by 12 (24\%) studies and ranged from 66-90\% attendance of exercise training sessions. Mean dropout from follow-up measurement was $10 \pm 10 \%$ in the 36 (72\%) studies in which attrition was clear. Adverse events were reported in 17 (34\%) studies. There were 18 musculoskeletal injuries attributable to the exercise interventions; 14/18 (72\%) occurred in the HIIT group. Injuries did not necessarily result in the affected participant having to drop out from the study or discontinue the intervention. No serious adverse events were reported (see Table S1). 
Data for fasting glucose, fasting insulin, $\mathrm{HbA1c}$, insulin resistance, $\mathrm{VO}_{2 \max }$ and body weight were included in the meta-analysis. Effect sizes for within groups and comparisons with CON and CT are presented in Table 1. Postprandial or post-challenge glucose levels were extracted but not analysed as there were not enough data to perform meaningful comparisons.

Insulin resistance Insulin resistance was estimated in 29 (58\%) studies. Of these, 20/29 (69\%) had at least one control group. The HOMA model was employed by 21/29 (72\%) studies. Other models of IR used were the QUICKI method $\left(n=1,3 \%{ }^{67}\right)$, Matsuda index $\left(n=4,14 \%^{37,52,68,69}\right)$, Cederholm index $\left(n=2,7 \%{ }^{70,71}\right)$ and the euglycaemic hyperinsulinaemic clamp $\left(n=1,3 \%{ }^{53}\right)$. There was a significant reduction in HOMA score of 0.33 (95\% CI -0.47 to $-0.18, \mathrm{p}<0.001$ ) with HIIT compared to baseline (Fig. S1). With all models of insulin resistance standardised for between group comparisons there was a significant reduction in insulin resistance compared to both CON and CT groups; Figure $2 a \& 2 b$.

Sensitivity analyses

When only studies using HOMA were included in pairwise comparisons, the standardised mean differences between HIIT and CT as well as HIIT and CON were somewhat attenuated; however, effects for HIIT versus CT remained significant (data not shown).

When studies were categorised by the time between final exercise session and post-test blood sample, we found that the improvement in insulin sensitivity diminished as the time after exercise increased (Fig S2).

Fasting glucose

Fasting glucose was reported in 47 (94\%) studies. Of these, 30/47 (64\%) were compared to at least one control group. There was a reduction in fasting glucose of $0.13 \mathrm{mmol} . \mathrm{L}^{-1}(-0.19$ to $-0.07, \mathrm{p}<0.001)$ with HIIT compared to baseline (Fig. S3), though this reduction was not different compared to the CON or CT groups overall (Figure $3 a \& 3 b$ ). Conversely, in those with metabolic syndrome or Type 2 diabetes, there was a reduction in fasting glucose of $0.92 \mathrm{mmol}^{-1} \mathrm{~L}^{-1}(-1.22$ to $-0.63, \mathrm{p}<0.001)$ following HIIT compared to CON (five studies; Figure 3a).

$\mathrm{HbA1c}$

Baseline and post-intervention HbA1c was reported by 13 (26\%) studies. Of these, 6/13 (46\%) had a CON group and 7/13 (54\%) had a CT group. Compared to baseline, there was no change in HbA1c (Fig.S4), however within the metabolic syndrome/Type 2 diabetes population there was a significant reduction of $0.25 \%$ ( -0.27 to $-0.23, \mathrm{p}<0.001)$. Similarly, there was no effect of HIIT compared to CON overall, but a significant reduction of $0.47 \%$ ( -0.92 to $-0.01, \mathrm{p}=0.04$ ) was observed in the metabolic syndrome/Type 2 diabetes group (Figure 4a). There was no change in HbA1c compared to CT overall, or within any of the population subgroups (Figure $4 b$ ). 


\section{Fasting insulin}

2 Fasting insulin was reported in 28 (56\%) studies. Of these, 19/28 (68\%) were compared to at least one

3 control group. There was a significant reduction in fasting insulin from baseline of $-0.93 \mu \mathrm{U} \cdot \mathrm{L}^{-1}(-1.39$ to -

$4 \quad$ 0.48, p<0.001; Fig S5.1) however, this effect was not present when HIIT was compared to a control group

$5 \quad$ (Fig S5.2 \& 5.3).

6 Body Weight

7 Studies reported body weight $(9 / 50 ; 18 \%)$, body mass index $(5 / 50 ; 10 \%)$ or both $(25 / 50 ; 48 \%)$. Of these,

8 23/34 (68\%; body weight) and 23/31 (74\%; body mass index) compared HIIT to at least one control group.

9 Compared to baseline, there was a $0.7 \mathrm{~kg}$ reduction in weight following HIIT (-1.19, -0.25, p=0.002; Fig.

10 S6.1). Compared to CON, the reduction was $1.3 \mathrm{~kg}(-1.90,-0.68, \mathrm{p}<0.001$; Fig S6.2). A greater effect of

$112.3 \mathrm{~kg}$ (-3.27 to -1.22, $\mathrm{p}<0.001)$ was observed in the metabolic syndrome/ Type 2 diabetes subgroup. In

12 contrast, there was no difference in weight loss following HIIT compared to CT overall (WMD $=0.32,-0.17$,

13 0.81, p=0.20; Fig S6.3). As expected, a similar pattern of changes were observed for BMI (data not shown).

14 Cardiorespiratory fitness

15 Cardiorespiratory fitness, expressed as $\mathrm{VO}_{2 \max }$, was reported in 42 (84\%) studies. Of these, 31/42 (74\%)

16 compared change in $\mathrm{VO}_{2 \max }$ to a control group. Compared to baseline, there was a 0.30L.min ${ }^{-1}$ increase in

$17 \mathrm{VO}_{2 \max }$ with HIIT (0.25 to 0.35, $\mathrm{p}<0.001$; Fig. S7.1). This increase was similar in comparison to CON

18 (WMD=0.28, 0.12 to 0.44 , p=0.001; Fig S7.2) and attenuated but still significant when compared to CT

19 (WMD=0.16, 0.07 to 0.25 , $\mathrm{p}=0.001$; Fig S7.3). 
Table 1 Effect sizes of comparisons of HIIT after training, compared to control and continuous training

\begin{tabular}{|c|c|c|c|c|}
\hline & & Within groups $^{\dagger}$ & Compared to CON & Compared to CT \\
\hline $\begin{array}{c}\text { Insulin } \\
\text { Resistance }\end{array}$ & $\begin{array}{c}\mathrm{N} \\
\text { ES (95\% CI) } \\
\mathrm{I}^{2}(\%) \\
\mathrm{p}\end{array}$ & $\begin{array}{c}23 \\
-0.33(-0.47,-0.18) \\
89.0 \\
<0.001\end{array}$ & $\begin{array}{c}11 \\
-0.49(-0.87,-0.12) \\
56.4 \\
0.009\end{array}$ & $\begin{array}{c}17 \\
-0.35(-0.68,-0.02) \\
58.7 \\
0.036\end{array}$ \\
\hline Fasting Glucose & $\begin{array}{c}\mathrm{N} \\
\text { ES (95\% CI) } \\
\mathrm{I}^{2}(\%) \\
\mathrm{p}\end{array}$ & $\begin{array}{c}47 \\
-0.13(-0.19,-0.07) \\
74.4 \\
<0.001\end{array}$ & $\begin{array}{c}18 \\
-0.17(-0.34,0.01) \\
67.8 \\
0.067\end{array}$ & $\begin{array}{c}23 \\
-0.07(-0.17,0.03) \\
4.9 \\
0.178\end{array}$ \\
\hline HbA1c & $\begin{array}{c}\mathrm{N} \\
\text { ES }(95 \% \mathrm{CI}) \\
\mathrm{I}^{2}(\%) \\
\mathrm{p} \\
\end{array}$ & $\begin{array}{c}13 \\
-0.13(-0.27,0.01) \\
99.0 \\
0.068 \\
\end{array}$ & $\begin{array}{c}6 \\
-0.19(-0.36,-0.03) \\
0.0 \\
0.021 \\
\end{array}$ & $\begin{array}{c}7 \\
0.02(-0.07,0.11) \\
18.5 \\
0.678 \\
\end{array}$ \\
\hline Fasting Insulin & $\begin{array}{c}\mathrm{N} \\
\text { ES (95\% CI) } \\
\mathrm{I}^{2}(\%) \\
\mathrm{p}\end{array}$ & $\begin{array}{c}28 \\
-0.93(-1.39,-0.48) \\
81.4 \\
<0.001\end{array}$ & $\begin{array}{c}11 \\
-1.0(-2.32,0.32) \\
57.5 \\
0.138\end{array}$ & $\begin{array}{c}16 \\
-0.34(-1.42,0.73) \\
0.0 \\
0.531\end{array}$ \\
\hline Body weight & $\begin{array}{c}\mathrm{N} \\
\text { ES (95\% CI) } \\
\mathrm{I}^{2}(\%) \\
\mathrm{p}\end{array}$ & $\begin{array}{c}34 \\
-0.72(-1.19,-0.25) \\
93.0 \\
0.002\end{array}$ & $\begin{array}{c}14 \\
-1.29(-1.90,-0.68) \\
21.4 \\
<0.001\end{array}$ & $\begin{array}{c}18 \\
0.32(-0.17,0.81) \\
33.2 \\
0.201\end{array}$ \\
\hline VO2max & $\begin{array}{c}\mathrm{N} \\
\text { ES (95\% CI) } \\
\mathrm{I}^{2}(\%) \\
\mathrm{p}\end{array}$ & $\begin{array}{c}44 \\
0.30(0.25,0.35) \\
97.9 \\
<0.001\end{array}$ & $\begin{array}{c}18 \\
0.28(0.12,0.44) \\
91.8 \\
0.001\end{array}$ & $\begin{array}{c}23 \\
0.16(0.07,0.25) \\
76.3 \\
0.001\end{array}$ \\
\hline
\end{tabular}

†Within groups effect sizes reflect the pooled difference before and after the intervention in the HIIT arm of each study including both controlled and non-controlled trials.

CON: non-exercising control; CT: continuous training; CI: confidence interval; N: number of studies included in analysis; ES: effect size; I²: study heterogeneity statistic; WMD: weighted mean difference; SMD: standardised mean difference 


\section{Meta-regression}

2 Table 2 shows the $§$ coefficients and confidence intervals for the regression analyses. HIIT characteristics;

3 interval intensity and weekly high-intensity exercise did not predict the improvements observed in insulin

4 resistance, fasting glucose, fasting insulin or HbA1c. Baseline levels of insulin resistance, fasting glucose

5 and fasting insulin predicted changes in these outcomes overall. Using the regression equation, we calculated

6 baseline insulin resistance would have to be $\geq 3.18$ to experience a reduction in HOMA-IR of -0.5 or greater.

7 Similarly, for a $0.1 \mathrm{mmol} . \mathrm{L}^{-1}$ or greater reduction in fasting glucose, baseline glucose would have to be

$8 \geq 4.92 \mathrm{mmol} . \mathrm{L}^{-1}$.When compared to non-exercising control groups, there was an inverse association between

9 baseline level and change in fasting glucose. Changes in body weight did not predict changes in insulin

10 resistance or glucose regulation. $\mathrm{VO}_{2 \max }$ was associated with a reduction in fasting glucose in studies that

11 included a non-exercising control group, however $\mathrm{VO}_{2 \max }$ did not predict other outcomes. 
Table 2. Meta-regression coefficients

\begin{tabular}{|c|c|c|c|c|c|c|c|}
\hline & & Interval Intensity & $\begin{array}{c}\text { Time at high- } \\
\text { intensity/week }\end{array}$ & Weeks" & Change in body weight & Baseline level & Change in $\mathrm{VO}_{2 \max }$ \\
\hline & & \multicolumn{6}{|c|}{ ß (95\% CI) } \\
\hline \multirow{3}{*}{$\begin{array}{c}\text { Insulin } \\
\text { Resistance }\end{array}$} & WG & $1.46(-12.97,15.88)^{\dagger}$ & $2.64(-10.63,15.92)^{\dagger}$ & $-27.71(-71.43,16.00)^{\dagger}$ & $0.10(-0.09,0.29)$ & $-0.22(-0.37,-0.06)^{*}$ & $0.00(-0.31,0.32)$ \\
\hline & CON & $8.19(-33.34,12.90)^{\dagger}$ & $-0.01(-0.02,0.03)$ & $-0.07(-0.17,0.03)$ & $0.35(-0.39,1.10)$ & $-0.24(-0.90,0.43)$ & $-0.09(-1.37,1.19)$ \\
\hline & $\mathrm{CT}$ & $2.73(-33.84,28.38)^{\dagger}$ & $8.00(-38.33,22.34)^{\dagger}$ & $-0.02(-0.13,0.09)$ & $0.27(-1.19,1.73)$ & $-0.10(-0.50,0.31)$ & $-0.72(-1.66,0.65)$ \\
\hline \multirow{3}{*}{$\begin{array}{l}\text { Fasting } \\
\text { Glucose }\end{array}$} & WG & $2.30(-3.90,0.54)^{\dagger}$ & $-1.65(-5.45,2.15)^{\dagger}$ & $-1.15(-15.02,12.72)^{\dagger}$ & $0.02(-0.07,0.10)$ & $-0.12(-0.22,-0.02)^{*}$ & $0.08(-0.20,0.36)$ \\
\hline & $\mathrm{CON}$ & $1.59(-12.50,15.68)^{\dagger}$ & $0.33(-13.71,14.38)$ & $-0.01(-0.03,0.02)$ & $0.09(-0.12,0.30)$ & $-0.29(-0.45,-0.12)^{*}$ & $-1.03(-1.89,-0.17)$ \\
\hline & $\mathrm{CT}$ & $-1.93(-4.86,8.72)^{\dagger}$ & $-0.54(-10.74,9.66)^{\dagger}$ & $-0.02(-0.07,0.03)$ & $-0.01(-0.13,0.12)$ & $-0.10(-0.24,0.03)$ & $-0.25(-0.58,0.07)$ \\
\hline \multirow{3}{*}{ HbA1c } & WG & $1.53(-12.31,15.37)^{\dagger}$ & $0.56(-11.31,12.43)^{\dagger}$ & $-0.54(-13.57,12.83)^{\dagger}$ & $-0.01(-0.08,0.06)$ & $-0.14(-0.31,0.03)$ & $0.25(-0.56,1.05)$ \\
\hline & $\mathrm{CON}$ & $-0.10(-1.21,1.01)$ & $0.02(-0.25,0.29)$ & $0.002(-1.12,0.12)$ & $0.02(-0.10,0.14)$ & $-0.13(0.56,0.30)$ & $-1.01(-4.40,2.38)$ \\
\hline & CT & - & - & - & $0.01(-0.21,0.22)$ & $-0.14(-0.38,0.10)$ & $0.42(-1.14,1.97)$ \\
\hline \multirow{3}{*}{$\begin{array}{l}\text { Fasting } \\
\text { Insulin }\end{array}$} & WG & $-0.01(-0.07,0.04)$ & $-0.01(-0.05,0.04)$ & $-0.15(-0.33,0.03)$ & $0.29(-0.41,1.00)$ & $-0.26(-0.42,-0.10)^{*}$ & $-0.36(-2.74,2.02)$ \\
\hline & $\mathrm{CON}$ & $-0.003(-0.11,0.10)$ & $0.02(-0.07,0.12)$ & $-0.21(-0.60,0.18)$ & $1.56(-0.06,3.17)$ & $-0.40(-0.87,0.08)$ & $8.34(-7.90,24.59)$ \\
\hline & $\mathrm{CT}$ & $0.003(-0.12,0.12)$ & $-0.05(-0.18,0.08)$ & $0.05(-0.37,0.46)$ & $0.71(-0.62,2.04)$ & $-0.29(-0.63,0.04)$ & $-0.77(-4.20,2.66)$ \\
\hline
\end{tabular}

WG: within groups; CON: non-exercising control; CT: continuous training; CI: confidence intervals

^: controlled for weekly high intensity duration and study length; $\bullet$ : controlled for interval intensity and study length; $:$ : controlled for interval intensity and weekly high-intensity duration; $\uparrow$ : these values have been multiplied by 1000 and therefore represent a per 1000 unit change in the independent variable; *: $p<0.05$; -: not enough data to perform this analysis 


\section{Discussion}

The results of this meta-analysis suggest that HIIT is effective at improving measures of insulin resistance compared to continuous exercise and a non-exercising control group. Importantly, the largest effects were seen in those with Type 2 diabetes or metabolic syndrome. Furthermore, in those with Type 2 diabetes or the metabolic syndrome there was a $0.92 \mathrm{mmol} . \mathrm{L}^{-1}$ reduction in fasting glucose and a $0.47 \%\left(5 \mathrm{mmol} . \mathrm{L}^{-1}\right)$ reduction in $\mathrm{HbA1c}$ when compared to studies with a non-exercising control group. Results for these measures and fasting insulin were less conclusive amongst the cohort as a whole and when compared to continuous exercise. There was a significant reduction of $1.3 \mathrm{~kg}$ in body weight compared to the nonexercising control group, an effect largely observed in those described as overweight, obese, with, or at risk of Type 2 diabetes. In addition, cardiorespiratory fitness improved compared to both controls, to an extent comparable with previous meta-analyses of HIIT interventions. ${ }^{22,27}$

The primary modifiable elements of HIIT protocols, defined here as interval intensity and weekly time spent at high-intensity, did not significantly alter intervention effectiveness in terms of insulin resistance, fasting glucose, HIbA1c or fasting insulin. Consistent with the results observed in the meta-analysis, those with the highest baseline values experienced the greatest benefits in insulin resistance and glucose regulation, although these associations were, largely, not present in controlled studies. Body weight and cardiorespiratory fitness both improved following HIIT, but changes in these outcomes did not tend to predict improvements in insulin resistance or glucose regulation. There was however, an inverse relationship between change in $\mathrm{VO}_{2 \max }$ and fasting glucose in controlled studies.

As far as we are aware this is the first systematic review and meta-analysis of the effects of HIIT on outcomes related to metabolic health. The findings extend the conclusions made by Adams ${ }^{30}$ who inferred that HIIT resulted in similar acute physiological adaptations as continuous training despite a lower energy expenditure. Here, we provide a quantified estimation of the training effects of HIT on insulin resistance, HbA1c, fasting glucose, fasting insulin and body weight.

\section{Clinical application}

Our study suggests that HIIT may reduce insulin resistance compared to both continuous exercise training and control conditions. Insulin resistance is a recognised precursor to Type 2 diabetes ${ }^{72}$ and has been identified as an independent risk factor for cardiovascular disease. ${ }^{73-75}$ We have shown that under supervised, laboratory conditions HIIT is effective in improving insulin sensitivity and potentially therefore improving glycaemic control and diabetes-related outcomes. Indeed, while data on HbA1c in this area are limited, the reduction following HIIT in those with or at risk of Type 2 diabetes in this study was $0.47 \%$ which is consistent with previous observations that report clinically significant reductions of up to $0.6 \%$ in HbA1c after a minimum of eight weeks of exercise training. ${ }^{2-4}$

Meta-regression results suggest that to achieve the observed reduction in HOMA-IR of 0.5 units, baseline HOMA needs to be at least 3.18, a value that has been consistently associated with the $50 \%$ most insulin 
resistant individuals within a population ${ }^{73,74}$ and indicating that HIIT may improve insulin sensitivity only in those who are insulin resistant. HIIT therefore has the potential to be used as an alternative therapeutic strategy to traditional physical activity interventions for those with or at risk of Type 2 diabetes.

\section{Potential mechanisms}

Improvement in peripheral insulin sensitivity is one of the main mechanisms that has been used to explain the enhancement in glycaemia following exercise training and has been widely demonstrated following both acute and chronic exercise training. ${ }^{76}$

Improvements in insulin sensitivity have often been associated with a reduction in body weight ${ }^{77}$. We found that HIIT reduced both insulin resistance and body weight, although meta-regression did not reveal an association between these two factors. This is congruous with the findings of Karstoft et al. ${ }^{78}$ who found that changes in body composition following HIIT explained less than $25 \%$ of improvements in insulin sensitivity in patients with Type 2 diabetes. However, we were unable to determine whether body composition or fat distribution were affected by HIIT. A reduction in abdominal adiposity - often achieved with exercise training $^{79}$ - may cause an improvement in hepatic insulin sensitivity, ${ }^{80}$ and it may be this that resulted in an improvement in HOMA-IR scores, rather than overall weight loss.

Furthermore, given the protective effect of cardiorespiratory fitness on $\mathrm{HbA} 1 \mathrm{c}^{81}$, morbidity ${ }^{82}$ and mortality ${ }^{83}$ in Type 2 diabetes, it is notable that change in $\mathrm{VO}_{2 \max }$ also did not predict changes in insulin resistance or glycaemic control in this study. It therefore appears that some adaptations associated with increased muscle oxidative capacity may be independent of those that promote metabolic health. Nonetheless, by providing evidence that HIIT may lead to greater reductions in insulin resistance than continuous exercise training, our study suggests that either the interval modality, or the greater exercise intensity facilitate benefits observed with continuous moderate-intensity exercise training. There are a number of established metabolic pathways that are likely to be enhanced by HIIT, with some support from recent investigations. These include skeletal muscle glucose uptake, ${ }^{84}$ GLUT-4 content ${ }^{85,86}$ and muscle glycogen depletion induced insulin sensitivity. ${ }^{71,87}$ Training adaptations have been associated with changes in body composition, muscle physiology $84-86,88,89$ and glucose metabolism ${ }^{90}$. There is some evidence that while muscle glycogen content is not greatly affected following moderate-intensity continuous activity lasting less than one hour, ${ }^{91}$ glycogen depletion is observed following vigorous-intensity exercise ${ }^{92}$ and is one way HIIT may enhance acute insulin sensitivity superior to moderate-intensity continuous exercise. ${ }^{71}$ It is unclear whether this acute response promotes chronic adaptations that enhance insulin sensitivity, although it is possible that repeated acute improvements may be as beneficial. ${ }^{93}$

The mechanisms that may be enhanced following HIIT compared to continuous exercise training need further elucidation as there is disagreement as to the optimum volume and intensity of exercise that stimulates the greatest benefits ${ }^{94,95}$ and which of these factors is more important in metabolic health. We found no relationship between exercise intensity or time spent at high-intensity and changes in 
glucose/insulin parameters meaning that we are unable to determine which characteristics of HIIT protocols induce the observed improvements in these outcomes. HIIT presents a unique challenge to optimising exercise prescription given the range of variables that can be manipulated. Some, ${ }^{42}$ but not all, ${ }^{95}$ studies suggest that exercise intensity is the primary factor determining the degree of metabolic adaptations, though these investigations have not assessed HIIT programmes specifically which, as discussed, introduces more nuanced exercise variables.

\section{Strengths and Limitations}

The strengths of this review include the comprehensive search strategy employed, the use of random effects meta-analysis and the focus on metabolic outcomes. Of note, none of the individual studies of metabolic syndrome or Type 2 diabetes patients reported a significant reduction in HbA1c compared to control, whereas the pooled effect showed one may occur following HIIT training. This demonstrates the advantages of meta-analysis and highlights the importance of conducting adequately powered trials.

However, this meta-analysis is not without limitation. Firstly, study quality was poor with only 4/36 (11\%) controlled studies deemed to have low risk of bias. Secondly, there was wide heterogeneity between participants, HIIT protocol, and intervention length as well as CT interventions making it difficult to generalise conclusions and make direct comparisons between HIIT and CT. This issue was addressed to the best of our ability by stratifying results by participant disease status and using meta-regression. Nonetheless, we highlight the need for more robust randomised controlled trials to be carried out in the future using standardised continuous training protocols. Thirdly, the length of time between the last bout of exercise and post-test blood samples was not reported by many of the studies measuring insulin resistance. This is important since we demonstrated that the improvement in HOMA-IR score diminished with increasing time to assessment. In addition it is possible that the use of HOMA-IR may underestimate the impact of HIIT on insulin sensitivity given that HOMA is more representative of hepatic insulin resistance ${ }^{41}$ and exercise is more likely to affect peripheral insulin resistance. ${ }^{96}$ Indeed, our sensitivity analysis indicated that this may have been the case in the included studies. It is also difficult to apply the reduction in HOMA-IR score found in this meta-analysis in a wider context and the clinical relevance of a change of -0.33 units is unclear.

The number of participants who underwent a HIIT intervention and who were likely to be insulin resistant represented just $23 \%$ of the study population. This could mean that the potential of HIIT to reduce insulin resistance is not fully illustrated by this study; as demonstrated by our meta-regression, and emphasises the need for more trials to be carried out in those at risk of or with Type 2 diabetes.

Despite the safety concerns associated with HIIT, few studies reported pre-screening results or adverse events. There were more exercise-related injuries reported in the HIIT interventions than control conditions, but it is difficult to draw conclusions from the limited data available. 
intensity physical activity performed three times per week. However, it is worth noting that in total, exercise sessions took $34 \pm 13$ minutes to complete (including warm-up, recovery intervals and cool down). It is important to elucidate whether the requirement to set aside 35 minutes three times per week to perform HIIT addresses the perceived barrier to physical activity of "lack of time".

\section{Suggestions for future research}

Our results suggest that HIIT per se has the potential to improve health outcomes, regardless of the precise protocol employed. However, it is clear that more studies should be conducted that compare the effects of HIIT to those of continuous training, particularly in people at risk of or with type 2 diabetes given these were where the strongest effects were observed. To this end studies should be of long enough duration and adequately powered to detect any potential changes in clinically relevant outcomes such as HbA1c. A greater understanding of the potential mechanisms stimulating the more potent effects of HIT compared to continuous training should be elucidated so they can be maximised through exercise training.

Just six studies included in this review were conducted in either a "free-living” or "real world" context. ${ }^{39,50 \text {, }}$ 61-63, 65 If HIIT is to be recommended to the general population it must be made practical and accessible. Interventions in community settings, requiring minimal specialist equipment and supervision should be conducted to assess uptake, adherence and compliance to the protocol. Few studies have measured effort and enjoyment of completing HIIT, with some positive responses, ${ }^{97-99}$ including in sedentary populations. ${ }^{100}$ The results should be extended to populations averse to exercise in order to determine whether HIIT would be taken up as a health promoting form of physical activity.

\section{Conclusions}

In conclusion, we have demonstrated that HIIT conveys benefits to metabolic health which in the instance of insulin resistance and $\mathrm{VO}_{2 \max }$ may be superior to the effect of traditional continuous training. HIIT may therefore be suitable as an alternative to continuous exercise training in the promotion of metabolic health and weight loss, particularly in those with Type 2 diabetes or the metabolic syndrome. However, given the identified limitations, more research is needed to determine both behavioural responses and clinical benefits over the longer term.

\section{Figure legends}

Figure 1 Study selection

Figure 2 Change in insulin resistance after HIIT compared to (a) control and (b) continuous training

Figure 3 Change in fasting glucose after HIT compared to (a) control and (b) continuous training

Figure 4 Change in HbA1c after HIT compared to (a) control and (b) continuous training

\section{Funding}

The primary author is being funded for a $\mathrm{PhD}$ in the Diabetes Research Centre, University of Leicester. 


\section{Contribution statement}

$2 \mathrm{CJ}$ and TY had the original idea for the review. CJ developed and revised the protocol with input from all 3 authors. CJ developed the search strategy, performed the searches and statistical analyses and wrote the first

4 draft of the article. CJ \& GO reviewed and extracted data. All authors contributed to the writing of the paper,

5 provided input throughout the study and approved the final manuscript.

\section{Copyright Statement}

The Corresponding Author has the right to grant on behalf of all authors and does grant on behalf of all

8 authors, a worldwide licence to the Publishers and its licensees in perpetuity, in all forms, formats and

9 media (whether known now or created in the future), to i) publish, reproduce, distribute, display and store

10 the Contribution, ii) translate the Contribution into other languages, create adaptations, reprints, include

11 within collections and create summaries, extracts and/or, abstracts of the Contribution, iii) create any other

12 derivative work(s) based on the Contribution, iv) to exploit all subsidiary rights in the Contribution, v) the

13 inclusion of electronic links from the Contribution to third party material where-ever it may be located; and,

14 vi) licence any third party to do any or all of the above. 
1 Daousi C, Casson IF, Gill GV, MacFarlane IA, Wilding JPH, Pinkney JH. Prevalence of obesity in type 2 diabetes in secondary care: association with cardiovascular risk factors. Postgrad Med J 2006; 82: 280-84.

2 Umpierre D, Ribeiro PA, Kramer CK, et al. Physical activity advice only or structured exercise training and association with HbA1c levels in type 2 diabetes: a systematic review and meta-analysis. JAMA. 2011; 305: 1790-9.

3 Boule NG, Haddad E, Kenny GP, Wells GA, Sigal RJ. Effects of exercise on glycemic control and body mass in type 2 diabetes mellitus - A meta-analysis of controlled clinical trials. JAMA. 2001; 286: 121827.

4 Gillies CL, Abrams KR, Lambert PC, et al. Pharmacological and lifestyle interventions to prevent or delay type 2 diabetes in people with impaired glucose tolerance: systematic review and meta-analysis. $B M J$. 2007; 334: 299.

5 Shaw K, Gennat H, O'Rourke P, Del C. Exercise for overweight or obesity. Cochrane Database of Systematic Reviews. 2006.

$6 \quad$ Franz MJ, VanWormer JJ, Crain AL, et al. Weight-loss outcomes: A systematic review and metaanalysis of weight-loss clinical trials with a minimum 1-year follow-up. J Am Diet Assoc. 2007; 107: 175567.

7 Sigal RJ, Kenny GP, Wasserman DH, Castaneda-Sceppa C, White RD. Physical activity/exercise and type 2 diabetes - A consensus statement from the American diabetes association. Diabetes Care. 2006; 29: 1433-38.

8 Albright A, Franz M, Hornsby G, et al. American College of Sports Medicine position stand. Exercise and type 2 diabetes. Med Sci Sports Exerc. 2000; 32: 1345-60.

$9 \quad$ Home P, Mant J, Diaz J, Turner C. Management of type 2 diabetes: summary of updated NICE guidance. BMJ. 2008; 336

10 Donnelly JE, Blair SN, Jakicic JM, Manore MM, Rankin JW, Smith BK. Appropriate Physical Activity Intervention Strategies for Weight Loss and Prevention of Weight Regain for Adults. Med Sci Sports Exerc. 2009; 41: 459-71.

11 Roth M. Self-reported physical activity in adults. In: R. Craig JM, and V. Hirani (ed.). Health Survey for England. National Centre for Social Research: London 2011; 21-43.

12 M Chaudhury DE. Accelerometry in adults. In: Craig R MJHV (ed.). Health Survey for England 2008. National Centre for Social Research: London 2011; 61-88.

13 Troiano RP, Berrigan D, Dodd KW, Masse LC, Tilert T, McDowell M. Physical Activity in the United States Measured by Accelerometer. Med Sci Sports Exerc. 2008; 40: 181-88.

14 Stutts WC. Physical activity determinants in adults. Perceived benefits, barriers, and self efficacy. AAOHN. 2002; 50: 499-507.

15 Koeneman MA, Verheijden MW, Chinapaw MJM, Hopman-Rock M. Determinants of physical activity and exercise in healthy older adults: A systematic review. Int J Behav Nutr Phys Activ. 2011; 8. 16 Korkiakangas EE, Alahuhta MA, Laitinen JH. Barriers to regular exercise among adults at high risk or diagnosed with type 2 diabetes: a systematic review. Health Prom Int. 2009; 24: 416-27.

17 Gibala MJ, Little JP, Macdonald MJ, Hawley JA. Physiological adaptations to low-volume, highintensity interval training in health and disease. $J$ Physiol. 2012; 590: 1077-84.

18 Gibala MJ. High-intensity interval training: a time-efficient strategy for health promotion? Curr Sports Med Rep. 2007; 6: 211-3.

19 Burgomaster KA, Hughes SC, Heigenhauser GJF, Bradwell SN, Gibala MJ. Six sessions of sprint interval training increases muscle oxidative potential and cycle endurance capacity in humans. $J$ Appl Physiol. 2005; 98: 1985-90.

20 Gist NH, Fedewa MV, Dishman RK, Cureton KJ. Sprint interval training effects on aerobic capacity: a systematic review and meta-analysis. Sports Med. 2013; 44: 269-79.

21 Sloth M, Sloth D, Overgaard K, Dalgas U. Effects of sprint interval training on VO2max and aerobic exercise performance: A systematic review and meta-analysis. Scand J Med Sci Sports. 2013; 23: e341-52. 22 Weston M, Taylor KL, Batterham AM, Hopkins WG. Effects of Low-Volume High-Intensity Interval Training (HIT) on Fitness in Adults: A Meta-Analysis of Controlled and Non-Controlled Trials. Sports Med. 2014; 44: 1005-17.

23 Hardcastle SJ, Ray H, Beale L, Hagger MS. Why sprint interval training is inappropriate for a largely sedentary population. Front Psych. 2014; 5: 1505.

19 
24 Rognmo O, Hetland E, Helgerud J, Hoff J, Slordahl SA. High intensity aerobic interval exercise is superior to moderate intensity exercise for increasing aerobic capacity in patients with coronary artery disease. Eu J Cardiovasc Prev Rehab. 2004; 11: 216-22.

25 Little JP, Gillen JB, Percival ME, et al. Low-volume high-intensity interval training reduces hyperglycemia and increases muscle mitochondrial capacity in patients with type 2 diabetes. $J$ Appl Physiol. 2011; 111: 1554-60.

26 Garber CE, Blissmer B, Deschenes MR, et al. Quantity and Quality of Exercise for Developing and Maintaining Cardiorespiratory, Musculoskeletal, and Neuromotor Fitness in Apparently Healthy Adults: Guidance for Prescribing Exercise. Med Sci Sports Exerc. 2011; 43: 1334-59.

27 Weston KS, Wisloff U, Coombes JS. High-intensity interval training in patients with lifestyleinduced cardiometabolic disease: a systematic review and meta-analysis. Br J Sports Med. 2013; 48: 122734.

28 Cornish AK, Broadbent S, Cheema BS. Interval training for patients with coronary artery disease: a systematic review. Eu J Appl Physiol. 2011; 111: 579-89.

29 Elliott AD, Rajopadhyaya K, Bentley DJ, Beltrame JF, Aromataris EC. Interval Training Versus Continuous Exercise in Patients with Coronary Artery Disease: A Meta-Analysis. Heart Lung Circ. 2015; 24: $149-57$.

30 Adams OP. The impact of brief high-intensity exercise on blood glucose levels. Diabetes Metab Syndr Obes. 2013; 6: 113-22

31 Moher D, Liberati A, Tetzlaff J, Altman DG. Preferred Reporting Items for Systematic Reviews and Meta-Analyses: The PRISMA Statement. Ann Intern Med. 2009; 151: 264-69.

32 Haskell WL. Health consequesnses of physical activity - understanding and challenges regarding dose-response. Med Sci Sports Exerc. 1994; 26: 649-60.

33 Higgins JPT, Altman DG, Gøtzsche PC, et al. The Cochrane Collaboration's tool for assessing risk of bias in randomised trials. BMJ. 2011; 18: 343.

34 Schjerve IE, Tyldum GA, Tjonna AE, et al. Both aerobic endurance and strength training programmes improve cardiovascular health in obese adults. Clin Sci. 2008; 115: 283-93.

35 Moreira MM, Souza HP, Schwingel PA, Sa CK, Zoppi CC. Effects of aerobic and anaerobic exercise on cardiac risk variables in overweight adults. Arq Bras Cardiol. 2008; 91: 200-6, 19-26.

36 Shaban N, Kenno KA, Milne KJ. The effects of a 2 week modified high intensity interval training program on the homeostatic model of insulin resistance (HOMA-IR) in adults with type 2 diabetes. $J$ Sports Med Phys Fitness. 2014; 54: 203-09.

37 Venables MC, Jeukendrup AE. Endurance training and obesity: Effect on substrate metabolism and insulin sensitivity. Med Sci Sports Exerc. 2008; 40: 495-502.

38 Higgins JPT, Green S (ed). Cochrane handbook for Systematic Reviews of Interventions (V5.1.0 [updated March 2011]. The Cochrane Collaboration, 2011. Available from www.cochrane-handbook.org.

39 Lunt H, Draper N, Marshall HC, et al. High intensity interval training in a real world setting: a randomized controlled feasibility study in overweight inactive adults, measuring change in maximal oxygen uptake. PLoS ONE. 2014; 9: e83256.

$40 \quad$ Boyd JC, Simpson CA, Jung ME, Gurd BJ. Reducing the Intensity and Volume of Interval Training Diminishes Cardiovascular Adaptation but Not Mitochondrial Biogenesis in Overweight/Obese Men. PLoS ONE. 2013; 8.

41 Matthews DR, Hosker JP, Rudenski AS, Naylor BA, Treacher DF, Turner RC. Homeostasis model assessment: insulin resistance and beta-cell function from fasting plasma glucose and insulin concentrations in man. Diabetologia. 1985; 28: 412-9.

42 Umpierre D, Ribeiro PAB, Schaan BD, Ribeiro JP. Volume of supervised exercise training impacts glycaemic control in patients with type 2 diabetes: a systematic review with meta-regression analysis. Diabetologia. 2013; 56: 242-51.

43 Palmer TM, Peters JL, Sutton AJ, Moreno SG. Contour-enhanced funnel plots for meta-analysis. Stata J. 2008; 8: 242-54.

44 Begg CB, Mazumdar M. Operating characteristics of a rank correlation test for publication bias. Biometrics. 1994; 50: 1088-101.

45 Egger M, Davey Smith G, Schneider M, Minder C. Bias in meta-analysis detected by a simple, graphical test. BMJ. 1997; 315: 629-34.

$46 \quad$ Swain DP, Leutholtz BC, King ME, Haas LA, Branch JD. Relationship between \% heart rate reserve and \% VO2 reserve in treadmill exercise. Med Sci Sports Exerc. 1998; 30: 318-21. 
$47 \quad$ Byrne NM, Hills AP. Relationships between HR and VO2 in the obese. Med Sci Sports Exerc. 2002; 34: 1419-27. secretion in healthy individuals. Metabolism. 2012; 61: 12-6.

49 Myers J, McAuley P, Lavie CJ, Despres J-P, Arena R, Kokkinos P. Physical Activity and Cardiorespiratory Fitness as Major Markers of Cardiovascular Risk: Their Independent and Interwoven Importance to Health Status. Prog Cardiovasc Dis. 2015; 57: 306-14.

50 Moholdt T, Bekken Vold M, Grimsmo J, Slordahl SA, Wisloff U. Home-based aerobic interval training improves peak oxygen uptake equal to residential cardiac rehabilitation: A randomized, controlled trial. PLOS ONE. 2012; 7.

51 Iellamo F, Caminiti G, Sposato B, et al. Effect of High-Intensity interval training versus moderate continuous training on 24-h blood pressure profile and insulin resistance in patients with chronic heart failure. Intern Emerg Med. 2014; 9: 547-52.

52 Cocks M, Shaw CS, Shepherd SO, et al. Sprint interval and endurance training are equally effective in increasing muscle microvascular density and eNOS content in sedentary males. J Physiol. 2013; 591: 64156.

53 Richards JC, Johnson TK, Kuzma JN, et al. Short-term sprint interval training increases insulin sensitivity in healthy adults but does not affect the thermogenic response to beta-adrenergic stimulation. $J$ Physiol. 2010; 588: 2961-72.

54 Babraj JA, Vollaard NB, Keast C, Guppy FM, Cottrell G, Timmons JA. Extremely short duration high intensity interval training substantially improves insulin action in young healthy males. BMC Endocr Disord. 2009; 9: 3.

55 Terada T, Friesen A, Chahal BS, Bell GJ, McCargar LJ, Boule NG. Feasibility and preliminary efficacy of high intensity interval training in type 2 diabetes. Diabetes Res Clin Pract. 2013; 99: 120-29.

56 Tjonna AE, Lee SJ, Rognmo O, et al. Aerobic interval training versus continuous moderate exercise as a treatment for the metabolic syndrome: A pilot study. Circulation. 2008; 118: 346-54.

57 Helgerud J, Hoydal K, Wang E, et al. Aerobic high-intensity intervals improve VO2max more than moderate training. Med Sci Sports Exerc. 2007; 39: 665-71.

58 Ciolac EG, Bocchi EA, Bortolotto LA, Carvalho VO, Greve JMD, Guimaraes GV. Effects of highintensity aerobic interval training vs. moderate exercise on hemodynamic, metabolic and neuro-humoral abnormalities of young normotensive women at high familial risk for hypertension. Hypertension Res. 2010; 33: 836-43.

59 Earnest CP, Lupo M, Thibodaux J, et al. Interval training in men at risk for insulin resistance. Int $J$ Sports Med. 2013; 34: 355-63.

60 Skleryk JR, Karagounis LG, Hawley JA, Sharman MJ, Laursen PB, Watson G. Two weeks of reduced-volume sprint interval or traditional exercise training does not improve metabolic functioning in sedentary obese men. Diabetes, Obes Metabol. 2013; 15: 1146-53.

61 Karstoft K, Winding K, Knudsen SH, et al. The effects of free-living interval-walking training on glycemic control, body composition, and physical fitness in type 2 diabetic patients: A randomized, controlled trial. Diabetes Care. 2013; 36: 228-36.

62 Morikawa M, Okazaki K, Masuki S, et al. Physical fitness and indices of lifestyle-related diseases before and after interval walking training in middle-aged and older males and females. BJSM. 2011; 45: 21624.

63 Madssen E, Arbo I, Granøien I, Walderhaug L, Moholdt T. Peak Oxygen Uptake after Cardiac Rehabilitation: A Randomized Controlled Trial of a 12-Month Maintenance Program versus Usual Care. PLOS ONE. 2014; 9: e107924.

64 Gunnarsson TP, Bangsbo J. The 10-20-30 training concept improves performance and health profile in moderately trained runners. J Appl Physiol. 2012; 113: 16-24.

65 Sandvei M, Jeppesen PB, Stoen L, et al. Sprint interval running increases insulin sensitivity in young healthy subjects. Arch Physiol Biochem. 2012; 118: 139-47.

66 Hwang CL, Yu CJ, Shih JY, Yang PC, Wu YT. Effects of exercise training on exercise capacity in patients with non-small cell lung cancer receiving targeted therapy. Support Care Cancer. 2012; 20: 316977.

67 Grieco CR, Swain DP, Colberg SR, et al. Effect of Intensity of Aerobic Training on Insulin Sensitivity/Resistance in Recreationally Active Adults. J Strength Cond Res. 2013; 27: 2270-76. 

of inflammatory and prominent proteomic changes in plasma and adipose tissue after high-intensity intermittent training in overweight and obese males. J Appl Physiol. 2012; 112: 1353-60.

69 Whyte LJ, Gill JMR, Cathcart AJ. Effect of 2 weeks of sprint interval training on health-related outcomes in sedentary overweight/obese men. Metabolism. 2010; 59: 1421-28.

71 Metcalfe RS, Babraj JA, Fawkner SG, Vollaard NBJ. Towards the minimal amount of exercise for improving metabolic health: Beneficial effects of reduced-exertion high-intensity interval training. Eur $J$ Appl Physiol. 2012; 112: 2767-75.

72 Alberti KG, Zimmet PZ. Definition, diagnosis and classification of diabetes mellitus and its complications. Part 1: diagnosis and classification of diabetes mellitus provisional report of a WHO consultation. Diabet Med. 1998; 15: 539-53.

73 Hanley AJG, Williams K, Stern MP, Haffner SM. Homeostasis Model Assessment of Insulin Resistance in Relation to the Incidence of Cardiovascular Disease: The San Antonio Heart Study. Diabetes Care. 2002; 25: 1177-84.

74 Bonora E, Formentini G, Calcaterra F, et al. HOMA-estimated insulin resistance is an independent predictor of cardiovascular disease in type 2 diabetic subjects - Prospective data from the Verona Diabetes Complicated Study. Diabetes Care. 2002; 25: 1135-41.

75 Kim J, Chae YK, Chernoff A. The Risk for Coronary Heart Disease According to Insulin Resistance with and without Type 2 diabetes. Endocr Rese. 2013; 38: 195-205.

76 Henriksen EJ. Exercise effects of muscle insulin signaling and action - Invited review: Effects of acute exercise and exercise training on insulin resistance. J Appl Physiol. 2002; 93: 788-96.

77 Goodpaster BH, Kelley DE, Wing RR, Meier A, Thaete FL. Effects of weight loss on regional fat distribution and insulin sensitivity in obesity. Diabetes. 1999; 48: 839-47.

78 Karstoft K, Winding K, Knudsen S, et al. Mechanisms behind the superior effects of interval vs continuous training on glycaemic control in individuals with type 2 diabetes: a randomised controlled trial. Diabetologia. 2014; 57: 2081-93.

79 Vissers D, Hens W, Taeymans J, Baeyens J-P, Poortmans J, Van Gaal L. The Effect of Exercise on Visceral Adipose Tissue in Overweight Adults: A Systematic Review and Meta-Analysis. PLoS ONE. 2013; 8.

80 Barzilai N, She L, Liu BQ, et al. Surgical removal of visceral fat reverses hepatic insulin resistance. Diabetes. 1999; 48: 94-98.

81 Boule NG, Kenny GP, Haddad E, Wells GA, Sigal RJ. Meta-analysis of the effect of structured exercise training on cardiorespiratory fitness in Type 2 diabetes mellitus. Diabetologia. 2003; 46: 1071-81. 82 Wei M, Gibbons LW, Mitchell TL, Kampert JB, Lee CD, Blair SN. The association between cardiorespiratory fitness and impaired fasting glucose and type 2 diabetes mellitus in men. Ann Intern Med. 1999; 130: 89-96.

83 Church TS, Cheng YJ, Earnest CP, et al. Exercise capacity and body composition as predictors of mortality among men with diabetes. Diabetes Care. 2004; 27: 83-88.

84 Etgen GJ, Brozinick JT, Kang HY, Ivy JL. Effects of exercise training on skeletal-muscle glucoseuptake and transport. Am J Physiol. 1993; 264: C727-C33.

85 Etgen GJ, Jensen J, Wilson CM, Hunt DG, Cushman SW, Ivy JL. Exercise training reverses insulin resistance in muscle by enhanced recruitment of GLUT-4 to the cell surface. Am J Physiol.1997; 272: E864E69.

$86 \quad$ Hood MS, Little JP, Tarnopolsky MA, Myslik F, Gibala MJ. Low-volume interval training improves muscle oxidative capacity in sedentary adults. Med Sci Sports Exerc. 2011; 43: 1849-56.

87 Bogardus C, Thuillez P, Ravussin E, Vasquez B, Narimiga M, Azhar S. Effect of muscle glycogen depletion on in-vivo insulin action in man. J Clin Invest. 1983; 72: 1605-10.

88 Kristiansen S, Hargreaves M, Richter EA. Exercise-induced increase in glucose transport, GLUT-4, and VAMP-2 in plasma membrane from human muscle. Am J Physiol. 1996; 270: E197-E201.

89 Kristiansen S, Hargreaves M, Richter EA. Progressive increase in glucose transport and GLUT-4 in human sarcolemmal vesicles during moderate exercise. Am J Physiol. 1997; 272: E385-E89.

$90 \quad$ Goodyear LJ, Kahn BB. Exercise, glucose transport, and insulin sensitivity. Annu Rev Med. 1998; 49: 235-61.

$91 \quad$ Kang J, Robertson RJ, Hagberg JM, et al. Effect of exercise intensity on glucose and insulin metabolism in obese individuals and obese NIDDM patients. Diabetes Care. 1996; 19: 341-49. 
92 Romijn JA, Coyle EF, Sidossis LS, Gastaldelli A, Horowitz JF, Endert E, et al. Regulation of endogenous fat and carbohydrate metabolism in relation to exercise intensity and duration. Am J Physiol. 1993; 265: E380-91.

93 Boule NG, Weisnagel SJ, Lakka TA, et al. Effects of exercise training on glucose homeostasis. Diabetes Care. 2005; 28: 108-14.

94 Colberg SR. The impact of exercise on insulin action in type 2 diabetes mellitus: Relationship to prevention and control. Insulin. 2006; 1: 85-98.

95 Houmard JA, Tanner CJ, Slentz CA, Duscha BD, McCartney JS, Kraus WE. Effect of the volume and intensity of exercise training on insulin sensitivity. J Appl Physiol. 2004; 96: 101-06.

96 Hawley JA, Lessard SJ. Exercise training-induced improvements in insulin action. Acta Physiol. 2008; 192: 127-35.

97 Bartlett JD, Close GL, MacLaren DPM, Gregson W, Drust B, Morton JP. High-intensity interval running is perceived to be more enjoyable than moderate-intensity continuous exercise: Implications for exercise adherence. $J$ Sports Scis. 2011; 29: 547-53.

98 Oliveira BRR, Slama FA, Deslandes AC, Furtado ES, Santos TM. Continuous and High-Intensity Interval Training: Which Promotes Higher Pleasure? PLOS ONE. 2013; 8.

99 Tritter A, Fitzgeorge L, Cramp A, Valiulis P, Prapavessis H. Self-efficacy and affect responses to Sprint Interval Training. Psychol Sport Exerc. 2013; 14: 886-90.

100 Jung ME, Bourne JE, Little JP. Where does HIT fit? An examination of the affective response to high-intensity intervals in comparison to continuous moderate- and continuous vigorous-intensity exercise in the exercise intensity-affect continuum. PLoS ONE. 2014; 9: e114541.

101 Adamson S, Lorimer R, Cobley JN, Lloyd R, Babraj J. High intensity training improves health and physical function in middle aged adults. Biology. 2014; 3: 333-44.

102 Harmer AR, A. Ruell P, et al. Effects of sprint training on extrarenal potassium regulation with intense exercise in Type 1 diabetes. J Appl Physiol. 2006; 100: 26-34.

103 Heydari M, Freund J, Boutcher SH. The effect of high-intensity intermittent exercise on body composition of overweight young males. J Obes. 2012; 2012.

104 Hovanloo F, Arefirad T, Ahmadizad S. Effects of sprint interval and continuous endurance training on serum levels of inflammatory biomarkers. J Diabetes Metab Disord. 2013; 12.

105 Keating SE, Machan EA, O'Connor HT, et al. Continuous Exercise but Not High Intensity Interval Training Improves Fat Distribution in Overweight Adults. J Obes. 2014; 2014: 12.

106 Mitranun W, Deerochanawong C, Tanaka H, Suksom D. Continuous vs interval training on glycemic control and macro- and microvascular reactivity in type 2 diabetic patients. Scand J Med Sci Sports. 2014; 24: e69-76.

107 Serpiello FR, McKenna MJ, Bishop DJ, et al. Repeated sprints alter signaling related to mitochondrial biogenesis in humans. Med Sci Sports Exerc. 2012; 44: 827-34.

108 Trapp EG, Chisholm DJ, Freund J, Boutcher SH. The effects of high-intensity intermittent exercise training on fat loss and fasting insulin levels of young women. Int J Obes. 2008; 32: 684-91.

109 Eguchi Y, Ohta M, Inoue T, et al. Effects of transitory stimulation interval exercise on physical function: a randomized controlled pilot study among Japanese Subjects. JUOEH. 2012; 34: 297-308.

110 Larsen S, Danielsen JH, Sondergard SD, et al. The effect of high-intensity training on mitochondrial fat oxidation in skeletal muscle and subcutaneous adipose tissue. Scand J Med Sci Sports. 2015; 25: e59-69.

111 Nybo L, Sundstrup E, Jakobsen MD, et al. High-intensity training versus traditional exercise interventions for promoting health. Med Sci Sports Exerc. 2010; 42: 1951-58.

112 Talanian JL, Holloway GP, Snook LA, Heigenhauser GJ, Bonen A, Spriet LL. Exercise training increases sarcolemmal and mitochondrial fatty acid transport proteins in human skeletal muscle. Am J Physiol. 2010; 299: E180-8.

113 Conraads VM, Pattyn N, De Maeyer C, et al. Aerobic interval training and continuous training equally improve aerobic exercise capacity in patients with coronary artery disease: The SAINTEX-CAD study. Int J Cardiol. 2015; 179: 203-10.

114 Fu TC, Wang CH, Lin PS, et al. Aerobic interval training improves oxygen uptake efficiency by enhancing cerebral and muscular hemodynamics in patients with heart failure. Int J Cardiol. 2013; 167: 4150 .

115 Heggelund J, Nilsberg GE, Hoff J, Morken G, Helgerud J. Effects of high aerobic intensity training in patients with schizophrenia-A controlled trial. Nord J Psychiatr. 2011; 65: 269-75. 
116 Hollekim-Strand SM, Bjorgaas MR, Albrektsen G, Tjonna AE, Wisloff U, Ingul CB. High-Intensity Interval Exercise Effectively Improves Cardiac Function in Patients With Type 2 diabetes Mellitus and Diastolic Dysfunction A Randomized Controlled Trial. J Am Coll Cardiol. 2014; 64: 1758-60.

117 Iellamo F, Manzi V, Caminiti G, Vitale C, Castagna C, Massaro M, et al. Matched dose interval and continuous exercise training induce similar cardiorespiratory and metabolic adaptations in patients with heart failure. Int J Cardiol. 2013; 167: 2561-65.

118 Madssen E, Moholdt T, Videm V, Wisloff U, Hegbom K, Wiseth R. Coronary Atheroma Regression and Plaque Characteristics Assessed by Grayscale and Radiofrequency Intravascular Ultrasound After Aerobic Exercise. Am J Cardio. 2014; 114: 1504-11.

119 Mora-Rodriguez R, Ortega JF, Hamouti N, et al. Time-course effects of aerobic interval training and detraining in patients with metabolic syndrome. Nutr Metabol Cardiovasc Dis. 2014; 24: 792-8.

120 Perry CG, Heigenhauser GJ, Bonen A, Spriet LL. High-intensity aerobic interval training increases fat and carbohydrate metabolic capacities in human skeletal muscle. Appl Physiol Nutr Metab. 2008; 33: 1112-23.

121 Stensvold D, Tjonna AE, Skaug EA, et al. Strength training versus aerobic interval training to modify risk factors of metabolic syndrome. J Appl Physiol. 2010; 108: 804-10.

122 Tjonna AE, Leinan IM, Bartnes AT, et al. Low- and High-Volume of Intensive Endurance Training Significantly Improves Maximal Oxygen Uptake after 10-Weeks of Training in Healthy Men. PLoS ONE.

$19 \quad 2013 ; 8$. 